\title{
Comportamientos sexuales y diferencias de género en adolescentes usuarios de un sistema público de salud universitario
}

\author{
Electra González $A^{a}$, Temístocles Molina $G^{b}$, Adela Montero, \\ Vania Martínez N, Carolina Leyton Mc. \\ Sexual behavior and gender differences \\ among adolescents consulting \\ at a university public health system
}

Background: The predetermined gender roles and the emphasis on reproductive responsibility on women, excluding men, have negative consequences on their sexual reproductive health and satisfactory sexual practices. Aim: To describe and analyze changes in sexual practices and gender differences in adolescents of the lower-middle socioeconomic level, users of public health system who started their sexual activity. Material and methods: We studied 4,971 adolescents of both sexes aged between 12 and 19 years, who consulted in a clinic for adolescents between the years 1990 and 2005. Several variables related to adolescent sexuality were considered. Uni and bivariate analysis were carried out and a model of stratified lineal regression per sex was fixed to explain the following variables: age at which sexual activity is initiated, number of sexual partners, time period between start of dating and the start of sexual activity along time. Results: The average age when men and women start their sexual activity was 15.7 and 15.5 years, respectively. Along years and among women but not men, there was a reduction in the age of start of sexual activity and an increase in the number of sexual partners. The mean lapse between start of dating and the start of sexual activity in men and women was 6.2 and 7.5 months, respectively. This figure had an $11.6 \%$ and $13.9 \%$ reduction per year of study in females and males, respectively. Conclusions: There is an increasing expansion of sexual roles in adolescents, but certain patterns of contradictory conservative reasoning are maintained (Rev Méd Chile 2007; 135: 1261-9).

(Key words: Adolescent; Gender identity; Sexual behavior; Sexuality)

Recibido el 28 de diciembre, 2006. Aceptado el 23 de abril, 2007.

Centro de Medicina Reproductiva y Desarrollo Integral de la Adolescencia, Facultad de Medicina, Universidad de Chile.

aAsistente Social, Máster en Investigación en Población

bEstadístico, Magister en Bioestadística

cPsicóloga Clínica Infanto Juvenil

Correspondencia a: Electra González A. Centro de Medicina

Reproductiva y Desarrollo Integral de la Adolescencia (CEMERA),

Facultad de Medicina, Universidad de Chile. Prof. Zañartu 1030,

Independencia, Santiago. Fax: 7356512.

E mail: cemera@med.uchile.cl 
$\mathrm{L}$ a sexualidad es principalmente la construcción social de un impulso biológico, que es además, multidimensional y dinámica. Es decir, la experiencia que una persona tenga de la sexualidad está mediada por la biología, los roles de género y las relaciones de poder, como también por factores tales como la edad y la condición social y económica. Pero la influencia social más profunda sobre la sexualidad de una persona proviene de los roles de género preestablecidos, las normas y valores sociales que determinan el poder relativo, las responsabilidades y las conductas de hombres y mujeres. Por lo tanto, la experiencia individual de la sexualidad no es más que la expresión de ese desequilibrio ${ }^{1,2}$.

Para los hombres, el rol histórico es la conquista sexual, como una forma de probar su propia masculinidad. Se estimula a los hombres a pensar en primer lugar en su desempeño sexual, por lo que el placer sexual de las mujeres se valora como una prueba del desempeño masculino, de lo contrario pueden ser identificados como «homosexuales». La homofobia es parte integral de la construcción social de la sexualidad masculina, y conduce a patrones de conducta tales como el inicio sexual temprano, muchas veces riesgoso, tener múltiples parejas o la actividad sexual coercitiva y abusiva. Desde esta perspectiva, la homofobia es destructiva para hombres y mujeres, ya sean homosexuales o heterosexuales ${ }^{1-5}$.

Para las mujeres, el rol prescrito es la pasividad en la actividad sexual, por lo tanto no se las alienta para que tomen decisiones respecto a la elección de sus parejas sexuales, para que negocien con sus compañeros el momento y la naturaleza de la actividad sexual, para que se protejan de un embarazo no deseado y de infecciones de transmisión sexual ${ }^{3-5}$.

Desde esta perspectiva, los roles de género que se refuerzan mutuamente, tienen consecuencias especialmente negativas para las prácticas sexuales satisfactorias y la salud sexual y reproductiva de hombres y mujeres ${ }^{5-8}$. Además, el énfasis en la responsabilidad del rol reproductivo de las mujeres excluye a los hombres, lo que significa que los hombres tienen un acceso limitado a servicios de salud reproductiva y su participación es mínima en la toma de decisiones responsables en este aspecto.

Es muy importante comprender que siendo la sexualidad una construcción social, se puede influir y modificar. El conversar, el encontrar un lenguaje para lo que no ha sido expresado, es una vía fundamental para que las personas modifiquen sus percepciones de sí mismas y para que lleguen a comprender la relación entre su comportamiento individual y el contexto social y cultural en que viven ${ }^{8-15}$

En la actualidad se observa una mayor liberalización de los comportamientos y actitudes sexuales y se manifiesta en forma especial en los adolescentes y, aunque se mantienen las diferencias entre los patrones de comportamiento sexual, hay una tendencia al acercamiento. Estudios nacionales muestran que las mujeres inician actividad sexual a los 17,8 años y los hombres a los 16,2 años, pero esta edad varía según grupo socioeconómico ${ }^{18}$. Hay una relación significativa entre edad de inicio sexual y nivel socioeconómico. Los jóvenes tienen relaciones sexuales en su mayoría con sus parejas, en especial las mujeres. El sexo ocasional ocurre más en los hombres. Se observa que 〈el amor» como motivo para tener relaciones sexuales ha ido disminuyendo a través de las diferentes encuestas, mientras que basta que ambos lo deseen» ha ido en aumento ${ }^{16-19}$.

Por otro lado, si bien el uso de métodos anticonceptivos ha aumentado en forma significativa, un porcentaje importante de jóvenes, especialmente en el estrato socioeconómico bajo y en sector rural, no los utiliza ${ }^{19}$.

Considerando que la edad de inicio de la actividad sexual, las características del comportamiento sexual y las diferencias de género en adolescentes son tópicos importantes a considerar en el diseño de programas de intervención para reducir el embarazo no deseado e infecciones de transmisión sexual (ITS) y VIH/SIDA, el objetivo de este estudio es describir y analizar los cambios en las prácticas sexuales y las diferencias de género en adolescentes de estratos socioeconómicos medio-bajo que iniciaron actividad sexual, usuarios de un sistema público de salud.

\section{MATERIAL Y MÉTODO}

Diseño de estudio transversal y analítico. El universo de estudio está constituido por adolescentes de ambos sexos, entre 12 y 19 años, que consultaron en el Centro de Medicina Reproductiva y Desamollo 
Integral de la Adolescencia, CEMERA de la Facultad de Medicina de la Universidad de Chile, entre los años 1990 y 2005. Una entrevista estructurada diseñada como instrumento para la atención de los y las adolescentes, y como instrumento de recolección de información para investigación, se les aplicó en la primera consulta. Los y las adolescentes firmaron un consentimiento informado donde se les explicó que los datos senán utilizados en investigación, protegiendo sus identidades. Fueron incluidos/as en el estudio sólo adolescentes que habían iniciado actividad sexual. Se excluyeron aquellos/as, en que el inicio de la actividad sexual fue por abuso sexual y aquellos/as que reportaron orientación sexual no heterosexual. Las variables estudiadas fueron: edad al momento de la entrevista, actividad, escolaridad (curso actual), rendimiento escolar (medido por promedio de notas del último año aprobado), proyectos académicos futuros, participación social (medido como pertenencia a alguna organización social ya sea deportiva, religiosa, artística u otra), edad al inicio de la relación afectiva (pololeo), edad al inicio de la actividad sexual coital, número de relaciones afectivas incluyendo pololeos o andar, número de parejas sexuales, tiempo transcumido entre pololeo e inicio de actividad sexual (medido en meses), contexto en que se dio el inicio de la actividad sexual, conocimiento y uso de métodos anticonceptivos y de prevención de infecciones de transmisión sexual (ITS) y VIH/SIDA. Para medir el nivel socioeconómico se aplico la escala de Graffar modificada.

Estadística. Los resultados para las variables (discretas y continuas) medidas en escala numérica, fueron expresados como valores promedios y desviación estándar y porcentaje para las medidas en escala nominal. Se realizaron análisis uni y bivariado aplicándose las pruebas estadísticas test $\mathrm{Chi}^{2}$, test diferencia de medias. Luego se ajustó un modelo de regresión lineal estratificado por sexo para explicar las variables edad de inicio de actividad sexual, número de parejas sexuales y tiempo transcumido entre inicio de pololeo e inicio de actividad sexual a través del tiempo. Se utilizaron los software EPI INFO 6.0, y STATA 8.0.

\section{RESUltados}

Los resultados corresponden a 4.971 adolescentes que habían iniciado actividad sexual. De éstos, 4.550
(91,5\%) eran mujeres y 421 (8,5\%) eran varones. Los y las adolescentes provenían de las diferentes comunas de la Región Metropolitana, exceptuando Vitacura, Las Condes, Providencia, La Reina, Ñunoa. El nivel socioeconómico fue medio-bajo. El promedio de edad de los varones a la entrevista fue de 17,4 años y de las mujeres, 16,3 años.

Eran estudiantes $90,3 \%$ de las mujeres y $69,7 \%$ de los varones. Cursaban $3^{\circ}$ y $4^{\circ}$ medio, $61,3 \%$ de los varones y $54,3 \%$ de las mujeres.

El rendimiento escolar alcanzó al 5,4 en hombres y mujeres.

El 37,3\% del total participaba en actividades sociales comunitarias, pero los varones lo hacían en mayor proporción que las mujeres, $65,6 \%$ versus $35,4 \%$. El tipo de participación social reportado fue, religiosas $26,7 \%$, deportivas $26 \%$ y recreativas artísticas $22,8 \%$. Al cruzar por sexo, los hombres en mayor proporción participan en actividades deportivas $50,8 \%$ versus $23,0 \%$, mientras que las mujeres participan más en actividades religiosas como artísticas recreativas, $28,6 \%$ y $23,7 \%$.

El 57,3\% del total tenía como aspiraciones académicas alcanzar estudios superiores. Al análisis por sexo, los varones con mayor frecuencia reportaron que querían realizar estudios superiores, $65,7 \%$ versus 56,8\%. (Tabla 1). El promedio de edad en eventos de sexualidad juvenil fueron para el inicio de relaciones afectivas de 13,6 años en hombres y 13,5 en mujeres, inicio de actividad sexual 15,7 años en hombres y 15,5 años en mujeres, siendo la diferencia promedio del tiempo entre inicio de la relación afectiva e inicio de actividad sexual: 6,2 meses para hombres y 7,5 meses para mujeres. Diferencias estadísticamente significativas para las cuatro últimas variables (Tabla 2). El 79,4\% del total de los y las adolescentes iniciaron actividad sexual con su actual pareja, al analizar por sexo, esta frecuencia fue mayor en las mujeres, $80,7 \%$ y $61,3 \%$, respectivamente. El 89,1\% del total inició actividad sexual con pololo/a, las mujeres en $90,3 \%$ y los hombres en $79,4 \%$. El motivo para iniciar actividad sexual fue en $41,4 \%$ por amor), en $28,2 \%$ se dio espontáneamente», en 15,9\% por curiosidad», en $12,9 \%$ fue una decisión conversada y planeada y sólo por retenerlo/a en 1,6\%. El sexo femenino reportó el amor en mayor proporción que los varones como la principal motivación para iniciar actividad sexual (42,8\% versus $30,1 \%)$, mientras que la categonáa «se dio espontáneamente»y por curiosi- 
Tabla 1. D istribución porcentual de variables personales de adolescentes por sexo (n: 4.971)

\begin{tabular}{|c|c|c|c|c|c|}
\hline Variables & Categorías & $\begin{array}{c}\text { Varones (421) } \\
\%\end{array}$ & $\begin{array}{c}\text { Mujeres (4.550) } \\
\%\end{array}$ & $\begin{array}{r}\text { Total } \\
\%\end{array}$ & $\mathrm{p}$ \\
\hline Actividad & $\begin{array}{l}\text { Estudia } \\
\text { Trabaja } \\
\text { Sin actividad }\end{array}$ & $\begin{array}{r}69,6 \\
5,7 \\
24,7\end{array}$ & $\begin{array}{r}90,3 \\
1,3 \\
8,4\end{array}$ & $\begin{array}{r}88,5 \\
1,7 \\
9,8\end{array}$ & 0,00 \\
\hline Escolaridad & $\begin{array}{l}\text { Básica } \\
\text { 1o a } 2^{\underline{0}} \text { Medio } \\
\text { 3o a } 4^{0} \text { Medio }\end{array}$ & $\begin{array}{l}10,5 \\
28,3 \\
61,3\end{array}$ & $\begin{array}{l}13,6 \\
32,1 \\
54,3\end{array}$ & $\begin{array}{l}13,3 \\
31,8 \\
54,9\end{array}$ & 0,01 \\
\hline $\begin{array}{l}\text { Participación } \\
\text { social }\end{array}$ & $\begin{array}{l}\text { Sí } \\
\text { No }\end{array}$ & $\begin{array}{l}65,6 \\
34,4\end{array}$ & $\begin{array}{l}35,4 \\
64,6\end{array}$ & $\begin{array}{l}37,3 \\
62,7\end{array}$ & 0,00 \\
\hline $\begin{array}{l}\text { Tipo } \\
\text { participación } \\
\text { social }\end{array}$ & $\begin{array}{l}\text { Deportivas } \\
\text { Religiosas } \\
\text { Recrea/artist } \\
\text { Comunitaria } \\
\text { Otros estudios } \\
\text { Trabaja }\end{array}$ & $\begin{array}{r}50,8 \\
12,6 \\
15,2 \\
5,2 \\
4,2 \\
12,0\end{array}$ & $\begin{array}{r}23,0 \\
28,6 \\
23,7 \\
5,8 \\
7,6 \\
11,3\end{array}$ & $\begin{array}{r}26,0 \\
26,7 \\
22,8 \\
5,7 \\
7,3 \\
11,3\end{array}$ & 0,00 \\
\hline $\begin{array}{l}\text { Aspiraciones } \\
\text { académicas }\end{array}$ & $\begin{array}{l}\text { E. superiores } \\
\text { E. media } \\
\text { Sin aspiraciones } \\
\text { académicas }\end{array}$ & $\begin{array}{r}65,7 \\
27,3 \\
7,0\end{array}$ & $\begin{array}{l}56,8 \\
30,2 \\
13,0\end{array}$ & $\begin{array}{l}57,3 \\
30,0 \\
12,6\end{array}$ & 0,00 \\
\hline
\end{tabular}

Tabla 2. D istribución porcentual de variables de sexualidad de adolescentes por sexo (n: 4.971)

\begin{tabular}{|c|c|c|c|c|c|}
\hline \multirow[t]{2}{*}{ Variables } & \multicolumn{2}{|c|}{ Varones (421) } & \multicolumn{2}{|c|}{ Mujeres (4.550) } & \multirow[t]{2}{*}{$\mathrm{p}$} \\
\hline & Promedio & Desv. Std. & Promedio & Desv Std. & \\
\hline $\begin{array}{l}\text { Edad inicio } \\
\quad \text { relación afectiva }\end{array}$ & 13,6 & 2,0 & 13,5 & 1,6 & 0,43 \\
\hline $\begin{array}{l}\text { Edad inicio } \\
\quad \text { actividad sexual }\end{array}$ & 15,7 & 1,7 & 15,5 & 1,4 & 0,02 \\
\hline $\mathrm{N}^{\circ}$ pololos/as & 4,2 & 3,9 & 3,3 & 2,8 & 0,00 \\
\hline $\mathrm{N}^{\circ}$ parejas sexuales & 1,9 & 1,8 & 1,3 & 0,9 & 0,00 \\
\hline $\begin{array}{l}\text { Tiempo de } \\
\text { relación afectiva al } \\
\text { iniciar actividad } \\
\text { sexual (meses) }\end{array}$ & 6,2 & 6,1 & 7,5 & 7,2 & 0,00 \\
\hline
\end{tabular}

dad» lo reportaron más frecuentemente los varones (Tabla 3).

El $95,2 \%$ de los y las adolescentes conocían la mayoría de los métodos anticonceptivos efectivos, (anticoncepción oral, inyectable, dispositivo intrauterino, condón), 78,5\% mencionó el condón masculino como método para prevención de infecciones de transmisión sexual y de VIH/SIDA y $55,4 \%$, señalaron al colegio como principal fuente de información, siendo más frecuente en mujeres. La mitad respondió que había usado previamente algún método anticonceptivo. El con- 
Tabla 3. D istribución porcentual de variables que rodea el inicio de la actividad sexual por sexo

\begin{tabular}{|c|c|c|c|c|c|}
\hline Variables & Categorías & $\begin{array}{r}\text { Varones } \\
\%\end{array}$ & $\begin{array}{r}\text { Mujeres } \\
\%\end{array}$ & $\begin{array}{r}\text { Total } \\
\%\end{array}$ & $\mathrm{p}$ \\
\hline $\begin{array}{l}\text { Pareja actual } \\
\text { inicio actividad } \\
\text { sexual }(\mathrm{n}=4.827)\end{array}$ & $\begin{array}{l}\text { Sí } \\
\text { No }\end{array}$ & $\begin{array}{l}61,3 \\
38,7\end{array}$ & $\begin{array}{l}80,7 \\
19,3\end{array}$ & $\begin{array}{l}79,4 \\
20,6\end{array}$ & 0,00 \\
\hline $\begin{array}{l}\text { Tipo relación } \\
\text { pareja con la que } \\
\text { inician actividad } \\
\text { sexual }(\mathrm{n}=2.128)\end{array}$ & $\begin{array}{l}\text { Pololo/a } \\
\text { Andante } \\
\text { Amigo/a } \\
\text { ocasional }\end{array}$ & $\begin{array}{r}79,4 \\
8,6 \\
5,6 \\
6,4\end{array}$ & $\begin{array}{r}90,3 \\
6,3 \\
2,3 \\
1,0\end{array}$ & $\begin{array}{r}89,1 \\
6,6 \\
2,7 \\
1,6\end{array}$ & 0,00 \\
\hline $\begin{array}{l}\text { Motivo para inicio } \\
\text { actividad sexual } \\
(\mathrm{n}=1.886)\end{array}$ & $\begin{array}{l}\text { Amor } \\
\text { Cedió al deseo } \\
\text { Curiosidad } \\
\text { Decisión ambos } \\
\text { Retenerlo/a }\end{array}$ & $\begin{array}{r}30,1 \\
31,1 \\
26,2 \\
11,7 \\
1,0\end{array}$ & $\begin{array}{r}42,8 \\
27,9 \\
14,6 \\
13,0 \\
1,7\end{array}$ & $\begin{array}{r}41,4 \\
28,2 \\
15,9 \\
12,9 \\
1,6\end{array}$ & 0,00 \\
\hline
\end{tabular}

dón masculino fue el método más reportado, siendo los varones quienes lo mencionaron más frecuentemente, $86,8 \%$ versus $67,4 \%$ (Tabla 4 ).

La edad de inicio de la actividad sexual en las adolescentes presentó una disminución progresiva a través del tiempo de 2,3\% por año (p: 0,00). Mientras que en los varones no presentó una tendencia clara $(p=0,70)$ (Figura 1). El número de parejas sexuales en las adolescentes presentó un aumento progresivo a través del tiempo de 1,7\% por año (p: 0,00), no observándose una tendencia clara en los varones ( $p=0,46)$ (Figura 2 ).

El tiempo de la relación afectiva al inicio de la actividad sexual presentó una disminución progresi-

Tabla 4. D istribución porcentual de conocimiento y práctica anticonceptiva por sexo

\begin{tabular}{|c|c|c|c|c|c|}
\hline Variables & Categorías & $\begin{array}{r}\text { Varones } \\
\%\end{array}$ & $\begin{array}{r}\text { Mujeres } \\
\%\end{array}$ & $\begin{array}{r}\text { Total } \\
\%\end{array}$ & $\mathrm{p}$ \\
\hline $\begin{array}{l}\text { Conoce mayoría métodos } \\
\text { anticonceptivos } \\
\text { efectivos }(\mathrm{n}=2.138)\end{array}$ & $\begin{array}{l}\text { Sí } \\
\text { No }\end{array}$ & $\begin{array}{r}94,8 \\
5,2\end{array}$ & $\begin{array}{r}95,2 \\
4,8\end{array}$ & $\begin{array}{r}95,2 \\
4,8\end{array}$ & 0,08 \\
\hline $\begin{array}{l}\text { Principal fuente } \\
\text { de información } \\
(\mathrm{n}=1.923)\end{array}$ & $\begin{array}{l}\text { Colegio } \\
\text { Padres } \\
\text { Amigos/as } \\
\text { Medios comunicación } \\
\text { Familiares }\end{array}$ & $\begin{array}{r}47,8 \\
15,9 \\
10,0 \\
17,4 \\
9,0\end{array}$ & $\begin{array}{r}56,3 \\
16,4 \\
10,5 \\
8,9 \\
7,9\end{array}$ & $\begin{array}{r}55,4 \\
16,4 \\
10,4 \\
9,8 \\
8,0\end{array}$ & 0,00 \\
\hline $\begin{array}{l}\text { Uso MAC } \\
\qquad(\mathrm{n}=2.455)\end{array}$ & $\begin{array}{l}\text { Condón } \\
\text { Píldora } \\
\text { Calendario } \\
\text { Inyectable }\end{array}$ & $\begin{array}{r}86,8 \\
6,1 \\
5,6 \\
1,5\end{array}$ & $\begin{array}{r}67,4 \\
17,5 \\
12,4 \\
2,7\end{array}$ & $\begin{array}{r}69,0 \\
16,6 \\
11,8 \\
2,6\end{array}$ & 0,00 \\
\hline
\end{tabular}




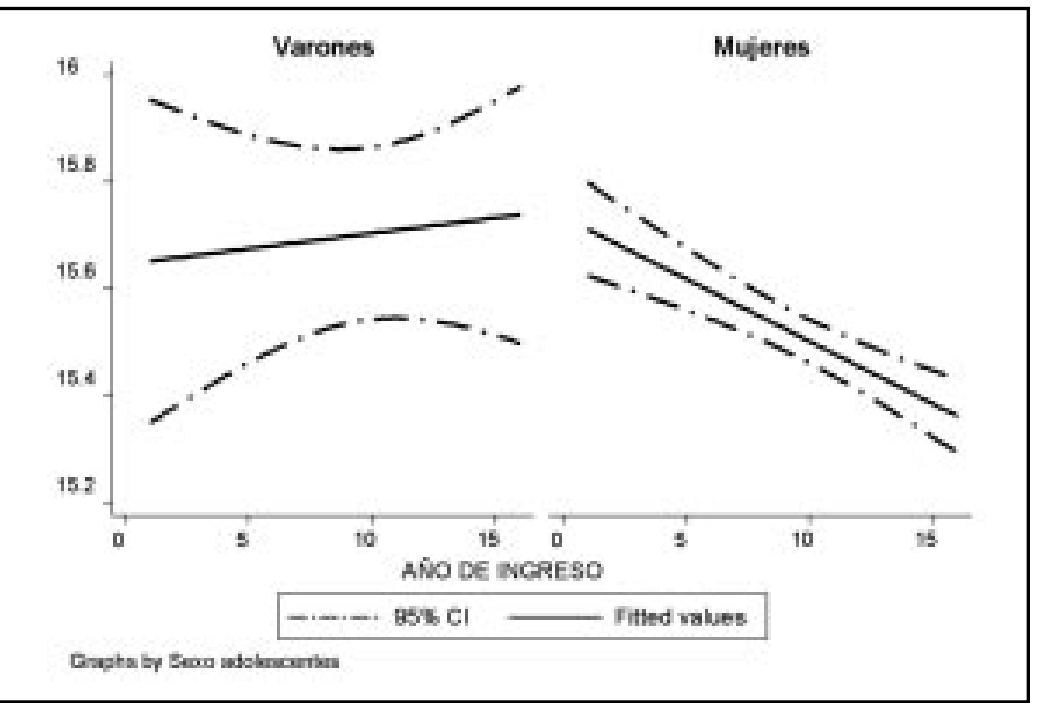

Figura 1. Edad inicio de actividad de sexual por año de ingreso y sexo.

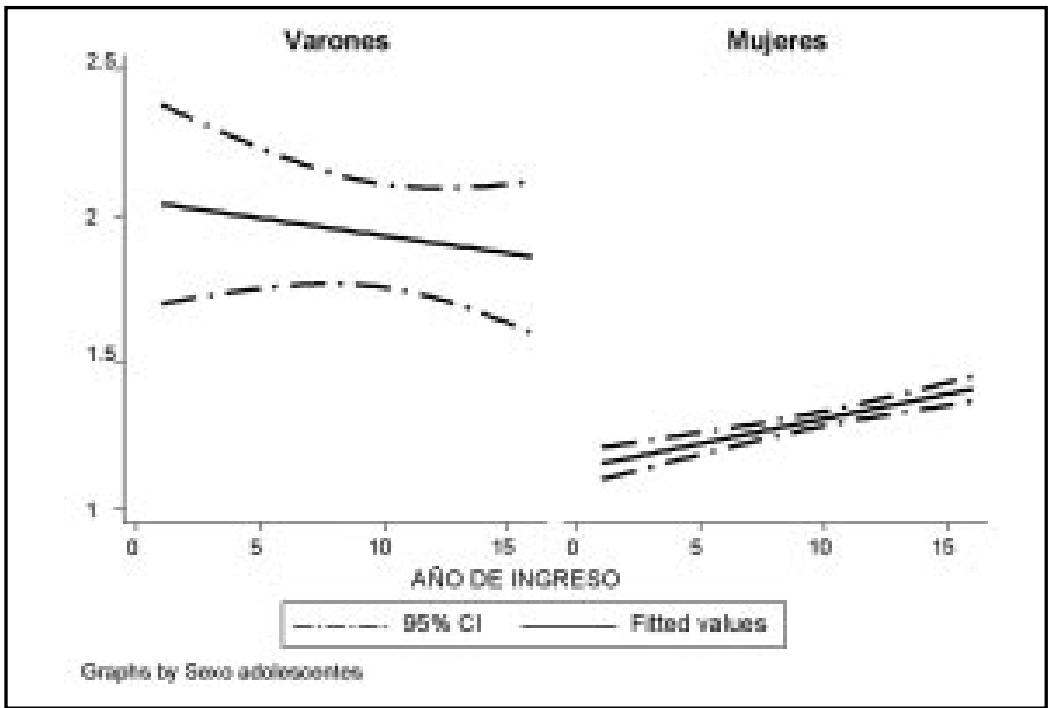

Figura 2. Número de parejas sexuales por año de ingreso y sexo.

va a través del tiempo de $11,6 \%$ en mujeres $(p=0,00)$ y de $13,9 \%$ en los varones ( $p=0,01$ ) (Figura 3 ).

\section{DisCUSIÓN}

La participación social de los y las adolescentes fue menor a la mostrada en los estudios del
Instituto Nacional de la Juventud, participando los varones en mayor proporción que las mujeres. $\mathrm{Al}$ analizar por tipo de participación, los hombres prefirieron las deportivas y las mujeres los grupos religiosos juveniles (catequista de primera comunión o de confirmación).

Este estudio mostró que el promedio de edad al inicio de la actividad sexual para adolescentes 


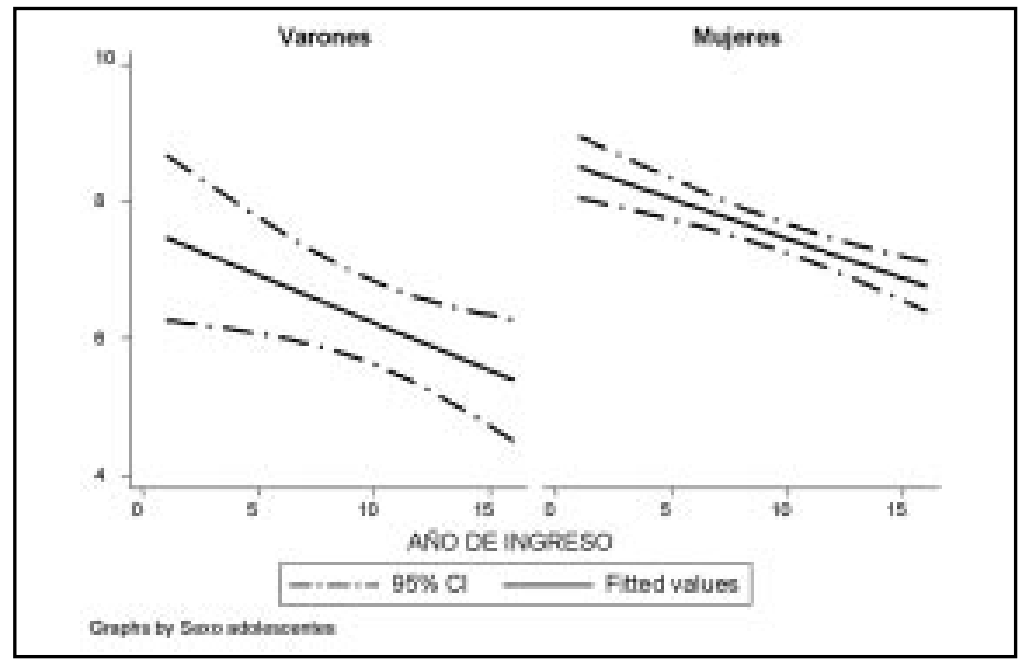

Figura 3. Tiempo de relación afectiva al iniciar actividad sexual (meses) por año de ingreso y sexo.

varones $(15,7)$ y adolescentes mujeres $(15,5)$ fue menor que lo reportado en otras investigaciones. Al estratificar por tiempo y sexo, se observó una disminución en la edad de inicio y un aumento en el número de parejas sexuales sólo en las mujeres. El tiempo promedio transcurrido entre el inicio de la relación afectiva y el inicio de la actividad sexual fue de 6,2 meses para los varones y 7,5 meses para las mujeres. Con una tendencia a disminuir de 11,6\% por año en las mujeres y de $13,9 \%$ en los varones. Estos son elementos importantes a considerar en la educación en sexualidad que imparte tanto la familia como el colegio.

Las adolescentes valoraron la sexualidad, el sexo y las relaciones sexuales en el contexto del amor romántico, donde principalmente estaban presentes tres elementos: que ésta se diera dentro de una pareja estable (pololeo), con un entorno romántico amoroso que incluya la ternura y el cariño, con otro requisito importante que es la monogamia y exclusividad, entendida la monogamia y la exclusividad mientras se está pololeando con esa persona, no para toda la vida. En este contexto, la noción de deseo y placer está directamente asociada y es aceptada en una pareja afectiva estable.

$\mathrm{Si}$ bien el mito del amor romántico, la pasividad erótica femenina siguen presente en el imaginario de $42,8 \%$ de las adolescentes entrevistadas, en las últimas décadas ha aparecido un nuevo patrón de adolescentes que integra más la sexualidad, que es más liberal. Por otro lado, «a prueba de amor»para retener al pololo, tan frecuentemente mencionada en estudios de décadas anteriores, tiene una incidencia muy baja en este estudio $(1,6 \%)$.

Conservar la virginidad hasta el matrimonio es un valor que muy pocas adolescentes tenían incorporado, aunque reconocieron que sí era un valor importante para sus padres, $89,1 \%$ del grupo en estudio inició su actividad sexual en una relación afectiva, frecuencia mayor, si lo comparamos con el 71,4\% de la última encuesta del INJUV.

Los «amigos/as con ventaja»y 〈andantes», tuvieron una menor incidencia, junto con «el sexo en carretes»en esta población, pero su frecuencia va en aumento. Por otra parte, en un tercio de los varones adolescentes ha aparecido un modelo de hombre más sensible, más vulnerable y que integra más el reconocimiento de las necesidades de la pareja.

Sólo la mitad de la población en estudio había utilizado un método anticonceptivo. Para las mujeres, la principal razón para no haber usado método anticonceptivo fue porque no podían ir al consultorio del sector debido a que sus padres no sabían de su actividad sexual. Para los y las adolescentes que habían usado un método anti- 
conceptivo previo a la consulta en CEMERA, encontramos que los más utilizados fueron el condón masculino (69\%) que pudieron adquirirlo en una farmacia y la anticoncepción oral (16,6\%), pero en la mayoría de las usuarias el uso fue irregular.

Es importante señalar que el condón fue utilizado principalmente como método anticonceptivo y no como medio de protección de infecciones de transmisión sexual y de VIH/SIDA. Al concurrir a CEMERA, los y las usuarios de condón solicitaron cambiar a otro método más seguro para prevención de embarazo.

Los resultados de este estudio permiten advertir una creciente ampliación de los roles sexuales de hombres y mujeres adolescentes, pero se mantienen pautas donde conviven discursos conservadores y transformadores en forma contradictoria. En este marco, aún se privilegia y valora una forma de relación entre hombres y mujeres como es la pareja heterosexual pero fuera del contexto de matrimonio y a edades cada vez más tempranas.

\section{REFERENCIAS}

1. Lamas M. La antropología feminista y la categoría de género. Nueva Antropología 1986; VIII, 30.

2. CAMPELL C. Male gender roles and sexuality: Implications for women's AIDS risk and prevention. Soc Sci Med 1995; 41: 197-210.

3. Santow G. Social roles and physical health: The case of female disadvantage in poor countries. Soc Sci Med 1995; 40: 147-61.

4. Learning about sexuality: a practical beginning, 1999, Zeidenstein Sondra y Moore Kirstein, Editors.

5. KANTNER JF, ZelniK M. Sexual experiences of young unmarried women in the U.S. Fam Plann Perspect 1972; 4: 9-18.

6. ZeLNIK M, KANTNER JF. Sexual and contraceptive experience of unmarried women in the United States, 1976 and 1971. Fam Plann Perspect 1977; 9: 55-71.

7. ZaBin LS, CLARK DS. Why the delay: A study of teenage family planning patients. Fam Plann Perspect 1981; 13: 205-17.
Entender los cambios en las prácticas sexuales y las diferencias de género, puede aumentar la eficacia de los programas de intervención diseñados para reducir embarazo no deseado e infecciones de transmisión sexual (ITS) y por VIH/SIDA en adolescentes de ambos sexos de estrato socioeconómico medio-bajo. Se deben concebir programas para los y las adolescentes activos sexualmente, pero no unidos en pareja todavía, y que aspiran a impedir embarazos y reducir su exposición a la transmisión de infecciones de transmisión sexual (ITS) y VIH/SIDA. Estos programas deberían combinar la educación, sensibilización y consejería, pues probablemente encontrarán resistencia en las fuerzas socioculturales imperantes actualmente, así como el ofrecimiento de medios de regulación de la fecundidad como un derecho en salud sexual y reproductiva. Los esfuerzos de los programas y de las intervenciones deberían estar focalizados en los grupos más vulnerables de la población, porque es allí donde los y las adolescentes suelen carecer de opciones vitales y oportunidades alternativas a los comportamientos reproductivos tempranos.

8. Zabin LS, Hirsch M, Smith E, HaRdy J. Adolescent sexual attitudes and behavior: Are they consistent. Fam Plann Perspect 1984; 6: 181-5.

9. FREEMAN L. Adolescent contraceptive use: Comparisons of male and female attitudes and information. Am J Public Health 1980; 70: 790-7.

10. Green V, Johnson S, Kaplan D. Predictors of adolescent female decision making regarding contraceptive usage. Adolescence 1992; 27: 61329.

11. Cvetrovich \& Grote. Psychosocial maturity and teenage contraceptive use: An investigation of decision-making and communication skills. Population and Environment 1980; 4: 211-26.

12. Golsmith A. Teenagers, sex and contraception. Fam Plann Perspect 1972; 4: 32-8.

13. HeRold N. Differences between women who begin pill use before and after first intercourse. Fam Plann Perspect 1980; 12: 304-5.

14. BAUMAN KE. Selected aspects of contraceptive practices of unmarried students. Med Aspect Hum Sex 1971; 5: 76-89. 
15. FurSTEnBerg F, SHEA J, Auson P. Contraceptive continuation among adolescents attending family planning clinics. Fam Plann Perspect 1983; 15: 211-17.

16. Instituto Nacional De La Juventud. Encuesta Nacional de Juventud. Chile, Informe Ejecutivo, Septiembre 2001.

17. Instituto Nacional de la Juventud. Tercera Encuesta Nacional de Juventud. La eventualidad de la inclusión: Jóvenes chilenos a comienzos del nuevo siglo, Gobierno de Chile, 2002.
18. Instituto Nacional de la Juventud. 4 ${ }^{\mathrm{a}}$ Encuesta Nacional de la Juventud 2003. Resultados Generales. 2004.

19. Instituto Nacional de la Juventud. Junio de 2006. Segundo Informe Nacional de Juventud.

20. CEPAL Adolescencia y Juventud en América Latina y el Caribe: Problemas, oportunidades y desafíos en el comienzo del nuevo siglo. Serie Población y Desarrollo. Año 2002. 